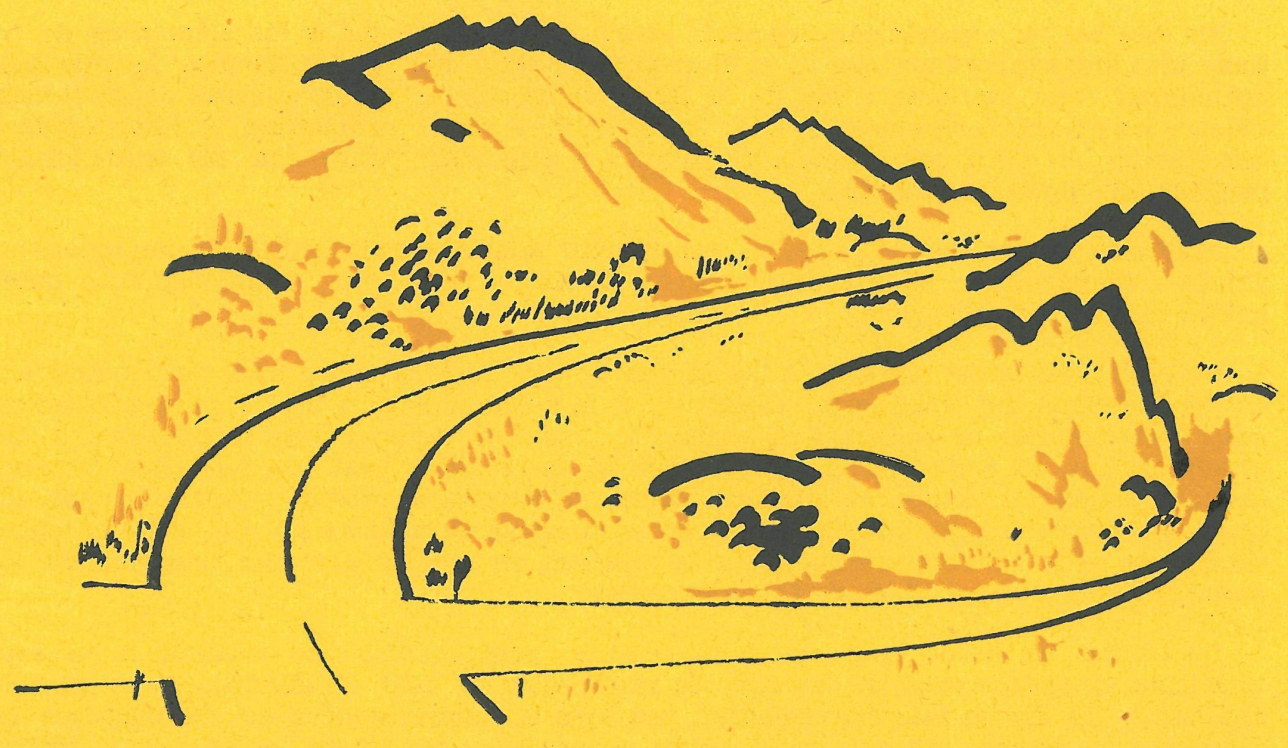

\title{
el problema financiero de las carreteras españolas
}

ANGEL VIZOSO MOZO, licenciado en ciencias económicas

Con mucha frecuencia se habla del "problema de nuestras carreteras" tratando de analizar el conjunto de causas que han producido el hecho de que se encuentren en un estado de rodaje verdaderamente deficiente, sobre todo si las comparamos con las de los restantes paises eurupeos, con los cuales más o menos tarde habrá de integrarse el nuestro, en cuyo caso uno de los aspectos a considerar, quizá previo a los demás, y desde luego muy importante, es el de la internacionalización de las carreteras, lo que supone la puesta a punto de las nuestras a un nivel similar al europeo, conforme a lo tratado en las recientes reuniones de los Ministros de Transporte europeos.

Por otro lado, la actual coyuntura económica española tiene encomendada a la etapa de "reactivación" que nos ocupa ahora, una vez conseguida la estabilización de precios, la imperiosa necesidad de exportar cada vez más productos españoles hacia los mercados mundiales, fundamentalmente hacia los países europeos y, principalmente, a los del Mercado Común, que presentan, sobre todo en 1960, un aumento muy notable en sus compras de mercancías españolas. De ello dan fe las últimas estadísticas del comercio exterior de España, de la Dirección General de Aduanas, según las cuales la C. E. E. (Comunidad Económica Europea) ha absorbido en los cuatro primeros meses del año 1960 casi el $50 \%$ de nuestras totales exportaciones, lo que representa un aumento del $250 \%$, respecto a igual periodo del año 1959, y es cuatro veces superior al incremento medio total. Atención, por tanto, a esta tendencia, puesto que si se mantiene esta importancia de "Ios seis" para nuestras exportaciones, quizá haya que buscar un acercamiento a este grupo, con el fin de que el establecimiento progresivo de su arancel aduanero externo y común no nos excluya paulatinamente, o al menos dificulte nuestras ventas a la C. E. E., sin fácil sustitución por otros mercados a donde exportar.

Es importante considerar este aumento de exportaciones españolas al Mercado Común, y el hecho de que cada vez se hará más imprescindible intensificar nuestra expansión comercial en este sentido-y en todos-, porque, según es sabido, el "estrangulamiento" fundamental de la economía española, el "bottle neck" en terminología americana e inglesa que impide nuestro rápido desenvolvimiento, reside sobre todo en la escasez de exportaciones españolas, lo que supone, a su vez, pocas posibilidades de importar, cuando ello es imprescindible (materias primas, maquinaria, etcétera, etc.) para que España deje de ser un "país subdesarrollado", según la terminología internacional, y se aumente en forma sustancial nuestro nivel medio de consumo. 
En esta mayor exportación española deben considerarse todos los elementos de coste y, sobre todo, uno importantísimo, que es el transporte, puesto que las condiciones del mercado mundial se aproximan cada vez más a las de la libre competencia, y las mismas asociaciones internacionales a las que pertenecemos (O. E. C. E., G. A. T. T., etc.) propugnan la libertad de intercambios comerciales, en pura competencia de precios y calidades, junto con las tradicionales armas de propaganda, presentación, etc.

Así, pues, el sumando "coste del transporte" adquiere extraordinaria importancia, por lo que vamos a ocuparnos de un aspecto concreto: el de las carreteras, como elemento determinante en buena medida de este coste.

No es el caso hacer mención a las causas de tipo histórico que han contribuído a la existencia de "el problema del transporte" español y a la conveniencia o no de su organización radial, con sus inconvenientes de retornos en vacío, etc., etc.; ni siquiera nos referiremos a dificultades de orden técnico, en virtud de las cuales todavía se recuerda casi con nostalgia los "firmes especiales" efectuados en tiempo del General Primo de Rivera. Hemos de ocuparnos aquí, sobre todo, del problema financiero, ya que en definitiva, si nuestras carreteras no son todo lo buenas que es de desear, se debe fundamentalmente a la penuria de medios con que ponerlas en las óptimas o mejores condiciones posibles de funcionamiento.

La técnica es, desde luego, muy importante; pero corresponde tratarla a los especialistas en la misma, ocupados también en algunos problemas de tipo económico, como pueden ser el del análisis de los firmes más convenientes, ventajas e inconvenientes de los elásticos basados en el asfalto, o los rígidos, de hormigón, según aconsejan y practican las modernas técnicas, especialmente en Alemania y otros países de Europa, análisis de velocidades, características geométricas de las carreteras, curvas, peraltes, etc., etc.

Trataremos el aspecto financiero, entendiendo que la solución de éste supone que los demás problemas técnicos pueden resolverse, mientras que de nada servirá tener realizados profundos estudios sobre la red ideal de autopistas españolas, por ejemplo, si luego no hay fondos con que llevarlas a la práctica.

Aclaremos que "solución financiera" quiere decir, para nosotros, algo más que el poder realizar inversiones suficientes, todo lo más, para efectuar nuevos riegos en el firme de las carreteras existentes, mejorar algo la señalización, retocar algunas curvas peligrosas, etc., etc., lo que supone obras con un plazo de vida limitado e insuficiente, sin atacar a fondo el problema, ya que tan sólo se consigue mantener nuestro circuito de carreteras en condiciones de rodaje durante breve tiempo.

Nuestra "solución financiera" sería mucho más cara y ambiciosa que la de un Plan que, como el presentado actualmente a las Cortes Españolas, totalice $\mathbf{8 0 . 0 0 0 ~ m i l l o n e s ~ d e ~ p e s e t a s ~ p a r a ~ d i e c i s e ́ i s ~}$ años, es decir, 5.000 millones de pesetas por año, sin tener en cuenta intereses, lo que realmente es, sobre todo, un plan de conservación, una campaña de bacheo intensivo, y algunas otras labores que de hecho no solucionarían en forma definitiva la puesta a punto de las carreteras españolas.

Por tanto, sigue siendo de importancia actual alguna solución financiera que incluso suplemente al Plan General, que debe considerarse como de urgencia y no como total y único para la puesta a punto de nuestras carreteras al nivel europeo, teniendo en cuenta, sobre todo, el volumen de inversión que realizan otros países, entre los cuales, a título de comparación, pueden citarse las siguientes cifras, según los datos de la "International Road Federation".

P A I S E S

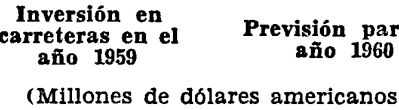

\begin{tabular}{|c|c|c|}
\hline Alemania Occidental $\ldots \ldots \ldots \ldots$ & 10.964 & 11.500 \\
\hline $\begin{array}{lllllllll}\text { Francia } & \ldots & \ldots & \ldots & \ldots & \ldots & \ldots & \ldots & \ldots\end{array}$ & 960 & 1.000 \\
\hline $\begin{array}{lllllllllll}\text { Italia } & \ldots & \ldots & \ldots & \ldots & \ldots & \ldots & \ldots & \ldots & \ldots\end{array}$ & 642 & 750 \\
\hline $\begin{array}{llllllllll}\text { Holanda } & \ldots & \ldots & \ldots & \ldots & \ldots & \ldots & \ldots & \ldots\end{array}$ & 320 & 350 \\
\hline Estados Unidos $\ldots \ldots \ldots \ldots \ldots$ & 65 & \\
\hline Argentina $\ldots \ldots \ldots \ldots \ldots$ & 51 & \\
\hline
\end{tabular}

Efectivamente, las inversiones efectuadas en las carreteras españolas no permitían, en los años precedentes, no ya solucionar el problema de su puesta en servicio en forma similar a las europeas, sino ni siquiera su mantenimiento en condiciones de circulación medianamente aceptables. Además, hay que tener en cuenta que, según ponen de manifiesto los trabajos de la Secretaría General Técnica del Ministerio de Obras Públicas, la inversión en carreteras ha venido representando, no sólo un porcentaje menor respecto a la inversión total que en la mayor parte de los países europeos, sino que, además, incluso llega a manifestar una tendencia decreciente, si se considera la inversión respecto a cada kilómetro de carretera. Las cifras resultan muy elocuentes y la pro- 
porción es tal, que si en Alemania se gastó en el año 1955 más de 180.000 pesetas por kilómetro, en España no llega a las 9.000 pesetas, cuando en Inglaterra sobrepasa las 29.000 , y en Bélgica las 40.000 , en Holanda 25.000 y en Portugal las 17.000, etc., etc.

Desde luego, el Estado debe atender con las disponibilidades existentes a la financiación de muy distintos sectores de la producción nacional, y su problema suele ser angustioso, sobre todo en momentos en que, como el actual, no se pueden tomar medidas que pueden producir nuevamente un proceso de inflación.

Por eso, sin duda, el Estado estimuló más otras inversiones, preferentemente las de producción minera e industrial, que las de servicios tales como transporte. Como consecuencia, los transportes y comunicaciones españolas sólo participaban, en el año 1955 y en conjunto, con un $5 \%$ en el producto interior bruto, porcentaje inferior a la de todos los países europeos, incluso los agrícolas mediterráneos, cuya media estaba en el $8 \%$.

La evolución de los transportes fué desde el año 1947 al 1957 muy retrasada con respecto a la producción minera e industrial; pero en los momentos actuales, en que se observa cierta laxitud o falta de reactivación en estos sectores, es probable que pueda atenderse, sobre todo con el Plan General de Carreteras en marcha, a una mejora sustancial en las nuestras, lo que realmente supone más favorables condiciones de nuestra estructura comercial, en el sentido de que mejores condiciones de transporte suponen menos tiempo de desplazamiento, menor coste y un efecto similar a la mejor calidad, principalmente para artículos de tipo agrícola que constituyen el porcentaje principal de la exportación española, que ha de dirigirse especialmente a los mercados europeos según anteriormente se indicaba.

A efectos de comparación se incluyen seguidamente las inversiones efectuadas en España para carreteras, desde los años 1942 al 1957, según los datos del Ministerio de Obras Públicas, cuyo detalle es el siguiente:

\begin{tabular}{|c|c|c|c|}
\hline$A$ N 0 & $\begin{array}{l}\text { Millones de pesetas } \\
\text { de cada año. } \\
\text { (Construcción, re- } \\
\text { paración y conser- } \\
\text { vaciốn.) }\end{array}$ & $\begin{array}{l}\text { En millones de pe- } \\
\text { setas del año } 1953 . \\
\text { (Inversión bruta en } \\
\text { carreteras, incluso } \\
\text { conservación.) }\end{array}$ & $\begin{array}{l}\text { Porcentaje respec- } \\
\text { to al producto na. } \\
\text { cional bruto. }\end{array}$ \\
\hline 1942 & 260,2 & 916 & 0,45 \\
\hline 1943 & 297,6 & 933 & 0,47 \\
\hline 1944 & 318,5 & 933 & 0,48 \\
\hline 1945 & 358,5 & 947 & 0,55 \\
\hline 1946 & 443,1 & 976 & 0,44 \\
\hline 1947 & 465,1 & 873 & 0,41 \\
\hline 1948 & 424,9 & 744 & 0,37 \\
\hline 1949 & 511,1 & 837 & 0,41 \\
\hline 1950 & 585,4 & 812 & 0,38 \\
\hline 1951 & 937,9 & 1.013 & 0,41 \\
\hline 1952 & $1.116,7$ & 1.195 & 0,46 \\
\hline 1953 & $1.116,7$ & 1.117 & 0,44 \\
\hline 1954 & $1.133,7$ & 1.128 & 0,40 \\
\hline 1955 & $1.133,7$ & 1.134 & 0,39 \\
\hline 1956 & $1.452,2$ & 1.275 & 0,42 \\
\hline 1957 & $1.452,2$ & 1.094 & 0,34 \\
\hline
\end{tabular}

Compárense las cifras de la cuarta columna, con la inversión bruta en carreteras, en porcentaje del producto nacional bruto de algunos países que a continuación se detallan:

Inversión bruta en \% del producto nacional bruto

\begin{tabular}{|c|c|}
\hline P A I S & $\begin{array}{c}\text { Transportes por } \\
\text { carretera, incluso } \\
\text { vehiculos particu- } \\
\text { lares. }\end{array}$ \\
\hline $\begin{array}{llllllllllll}\text { España } & \ldots & \ldots & \ldots & \ldots & \ldots & \ldots & \ldots & \ldots & \ldots & \ldots & \ldots\end{array}$ & 1,0 \\
\hline $\begin{array}{llllllllll}\text { Luxemburgo } & \ldots & \ldots & \ldots & \ldots & \ldots & \ldots & \ldots & \ldots & \ldots\end{array}$ & 2,9 \\
\hline 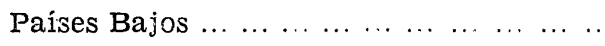 & 3,3 \\
\hline Alemania Occidental $\ldots \ldots \ldots c c c c c$ & 2,8 \\
\hline $\begin{array}{llllllllllll}\text { Bélgica } & \ldots & \ldots & \ldots & \ldots & \ldots & \ldots & \ldots & \ldots & \ldots & \ldots & \ldots\end{array}$ & $2,7-2,9$ \\
\hline $\begin{array}{llllllllllll}\text { Francia } & \ldots & \ldots & \ldots & \ldots & \ldots & \ldots & \ldots & \ldots & \ldots & \ldots & \ldots\end{array}$ & 2,8 \\
\hline $\begin{array}{lllllllllllll}\text { Italia } & \ldots & \ldots & \ldots & \ldots & \ldots & \ldots & \ldots & \ldots & \ldots & \ldots & \ldots & \ldots\end{array}$ & 2,8 \\
\hline
\end{tabular}


Para completar esta visión de las inversiones en carreteras, es interesante mencionar los cálculos preparados por el Gabinete de Planificación y Estudios Económicos del Ministerio de Obras Públicas, sobre las inversiones precisas desde el año 1958 hasta el 1970, bajo la base de que la tasa de crecimiento anual "per capita", sea del 3 y del $5 \%$. En estos supuestos se obtendrían las siguientes cifras de inversión:

\begin{tabular}{|c|c|c|c|c|}
\hline \multirow{2}{*}{$A \times 0$} & \multicolumn{2}{|c|}{$\begin{array}{l}\text { Renta prevista } \\
\text { (dólares por habitante) }\end{array}$} & \multicolumn{2}{|c|}{$\begin{array}{c}\text { Inversión necesaria en carretera } \\
\text { (millones de pesetas) }\end{array}$} \\
\hline & Supuesto aumento & $\begin{array}{c}\text { Supuesto a umento } \\
3 \%\end{array}$ & $\begin{array}{l}\text { Supuesto aumento } \\
5 \%\end{array}$ & $\underset{3 \%}{\text { Supuesto aumento }}$ \\
\hline 1958 & 298 & 286 & 3.243 & 3.113 \\
\hline 1959 & 313 & 295 & 3.557 & 3.276 \\
\hline 1960 & 328 & 304 & 3.901 & 3.449 \\
\hline 1961 & 345 & 313 & 4.297 & 3.630 \\
\hline 1962 & 462 & 322 & 4.693 & 3.821 \\
\hline 1963 & 380 & 332 & 5.147 & 4.023 \\
\hline 1964 & 399 & 342 & 5.645 & 4.234 \\
\hline 1965 & 419 & 352 & 6.192 & 4.457 \\
\hline 1966 & 440 & 363 & 6.791 & 4.692 \\
\hline 1967 & 462 & 374 & 7.448 & 4.939 \\
\hline 1968 & 485 & 385 & 8.169 & 5.199 \\
\hline 1969 & 509 & 397 & 8.960 & 5.472 \\
\hline 1970 & 535 & 408 & 9.827 & 5.760 \\
\hline
\end{tabular}

Como se ve por las cifras y datos anteriores, las inversiones españolas en carreteras han venido siendo muy insuficientes, y, por lo tanto, dentro de su escasez manifiesta, el Plan General de Carreteras presentado a las Cortes prevé un suplemento de asignación presupuestaria de $1.600 \mathrm{mi}-$ llones de pesetas anuales, lo que representa un aumento del $80 \%$ del presupuesto vigente, que permitirá indudablemente obras de señalización, conservación, consolidación y mejoras de firmes, muy importantes dada la angustiosa situación actual, que, según frases del propio Director de Carreteras, mantenía las nuestras en un estado "próximo al de ruina".

Tengamos en cuenta, no obstante, que, como muy bien ha dicho un Ministro italiano de Transportes, "somos un país pobre, tan pobre, que no podemos permitirnos el lujo de tener malas carreteras": frase perfectamente aplicable a España. Así como aquella otra de Colbert, de que "el progreso y el bienestar público dependen, principalmente, de la facilidad de los caminos".

Por ello, hay que señalar que todavía se hace necesario un plan más ambicioso, que quizá quepa dentro del nuevo cauce que el Plan General presentado a las Cortes abre a la iniciativa privada, corporaciones públicas, organismos autónomos, e incluso a los capitales extranjeros, que, conforme al Proyecto de Ley presentado, podrán participar con sus inversiones y dentro de las normas de regulación vigentes en esta materia, de fecha 26 de julio del año 1959, en la mejora de las carreteras españolas, tema del que también se ocupará en cuanto a financiación, la Comisión de Expertos del Banco Mundial de Reconstrucción y Desarrollo, al que pertenecemos, y que con este fln se desplazará a España próximamente.

Porque, evidentemente, si el Plan General presente puede mejorar notablemente nuestras carreteras a largo plazo, los problemas de posible incorporación en el futuro al Mercado Común Europeo, y en todo caso el aumento sustancial de nuestras exportaciones mediante el transporte terrestre a estos sectores, según se apuntaba al principio en este trabajo, implicará la urgencia de una Red de carreteras muy superior a la actual, de acuerdo también con la internacionalización prevista para los transportes, y con la necesidad de atender a los modernos sistemas de circulación en carreteras, quizá con el establecimiento de un sistema de autopistas, cuyo porvenir parece fuera de toda duda según vaya aumentando el tráfico, y que plantea problemas flnancieros de otra escala muy superior.

Atención, pues, a la financiación de carreteras españolas, necesitadas de un Plan General, como el presentado a las Cortes, y de otro de estructuración definitiva, de cara a un futuro muy inmediato, incluso con el apoyo de las organizaciones internacionales de crédito, capital extranjero, etc., con el que pueda pensarse en soluciones más caras (autopistas de hormigón, etc.), pero quizás más "económicas" a largo plazo. Pero de ello nos ocuparemos próximamente. 